
estratégica de recursos humanos: Efectiva forma de identificar necesidades de personal. Económicas CUC, 37(1), 61-78. DOI: http://dx.doi.org/10.17981/econcuc.37.1.2016.03

\title{
Planificación estratégica de recursos humanos: efectiva forma de identificar necesidades de personal
}

DOI: http://dx.doi.org/10.17981/econcuc.37.1.2016.03

\author{
Darcy Mendoza Fernández ${ }^{1}$ \\ Danny López Juvinao ${ }^{2}$ \\ Edwin Salas Solano ${ }^{3}$
}

\begin{abstract}
Resumen
El propósito del presente artículo es reflexionar sobre cómo la necesidad de supervivencia de toda organización, convoca la utilización de instrumentos como la planificación estratégica de recursos humanos para que de una forma proactiva puedan enfrentarse a las actuaciones empresariales en contexto globales, permitiéndoles de esta forma prever el futuro y salir avante con sus estrategias. La metodología utilizada es de tipo documental, a través de la cual se realizó una revisión bibliográfica que permitió el análisis de fundamentos y constructos teóricos. Los resultados obtenidos, se condensan en que la planificación estratégica de recursos humanos es necesaria desde cualquier punto de vista, puesto que aportan beneficios sustanciales a la gestión institucional al definir en horizontes en el corto y largo plazo en el avance y las competencias necesarias del personal para contribuir al logro de los objetivos estratégicos de la organización.
\end{abstract}

Palabras clave: planificación estratégica; gestión de recursos humanos; detección de necesidades de recursos humanos.

Recibido: 23.9.2015 Devuelto para revisión: 27.10.2015 Aceptado: 1.12.2015

\begin{abstract}
${ }^{1}$ Doctora en Ciencias Gerenciales. Magister Gerencia Empresarial. Universidad Rafael Belloso Chacín, Venezuela. Especialista en Finanzas. Universidad de la Costa-Barranquilla, Colombia. Administrador de empresas. Universidad de La Guajira-Colombia. Docente de planta adscrita al Grupo de Investigación E’ITAJAA, Universidad de La Guajira-Colombia. Autor de correspondencia, dmendoza@uniguajira.edu.co.
\end{abstract}

${ }^{2}$ Doctor en Ciencias Gerenciales. Magister en Gerencia empresarial. Universidad Rafael Belloso Chacín. Venezuela. Ingeniero en Minas. Universidad Pedagógica y Tecnológica de Colombia. Docente de planta adscrito al grupo de investigación Contacto con la comunidad. Universidad de La Guajira. Colombia.dlopezj@uniguajira.edu.co

${ }^{3}$ Candidato a Doctor en Ciencia Mención Gerencia. Universidad Rafael Bellos Chacín. Venezuela. Magister en Tributación y Política Fiscal. Universidad de Medellín-Colombia. Especialista en Gerencia en Finanzas. Contador Público. Universidad de la GuajiraColombia. Docente adscrito al Grupo de Investigación E’ITAJAA. Universidad de la Guajira, Colombia. esalas@uniguajira.edu.co.

o The author; licensee Universidad de la Costa - CUC.

Económicas CUC vol. 37 no. 1, pp. 61-78. Enero - Junio, 2016 Barranquilla. ISSN 0120-3932 Impreso, ISSN 2382-3860 Online 


\title{
Strategic Planning of Human Resources: Effective Way to Identify Personnel Needs
}

\begin{abstract}
The purpose of this article is to reflect on how the need for survival of any organization calls for the use of tools such as strategic planning of human resources so that they can proactively deal with business actions at a global context, enabling them to anticipate the future and to get ahead with their strategies. The methodology used is documentary, through a carried out bibliographic review, which allowed the analysis of foundations and theoretical constructs. The results obtained, are summarized that the strategic planning of human resources is necessary from any point of view, given that they contribute substantial benefits to the institutional management by defining in short and long term horizons the level and the necessary skills of staff to contribute in the achievement of the organization's strategic objectives.
\end{abstract}

Keywords: strategic planning; human resources management; detection of human resources needs.

\section{Introducción}

Hoy en día las organizaciones modernas, tanto públicas como privadas, operan en un entorno en donde el cambio es constante y permanente. La necesidad de supervivencia de toda organización hace que instrumentos como la planificación estratégica de recursos humanos sea para ellas una forma proactiva para poder enfrentarse a las acciones organizacionales, de tal manera que le permita de una u otra forma prever el futuro y salir avante con sus estrategias.

Las organizaciones son entes que generan sus propios objetivos y diseñan la forma como alcanzarlos teniendo en cuenta, necesariamente, las influencias del entorno social, político y económico. Son sistemas abiertos influenciados por el exterior. Estas influencias deben ser armonizadas internamente para que trabajen de forma coordinada en la consecución de los objetivos de la misma. De allí que, en las organizaciones se hace preciso introducir una gestión estratégica como una preocupación por el entrono. Este proceso define la misión y objetivos esenciales de la organización en relación con su entorno para operar sobre los elementos estratégicos de la misma, con la finalidad de cumplir los objetivos. Surge entonces lo que es la planificación 
estratégica del recurso humano que establece el número y tipo de gente adecuada que está disponible para realizar las actuaciones que resultarán de máximo aprovechamiento para la organización.

En este sentido, el presente artículo muestra la forma cómo se debe implementar la planificación estratégica del recurso humano, la cual contempla varias fases, como son: la definición de los objetivos del recurso humano, el estudio de la situación actual, el análisis del desfase, la elaboración de los planes estratégicos, alternativos y funcionales, y por último, la implementación y evaluación del proceso que contempla la definición de la plantilla de manera cuantitativa y cualitativa, culminando así en la elaboración de un plan de carrera.

A modo de síntesis, se puede decir que la planificación de los recursos humanos es una actividad propia de las organizaciones que alcanza su máxima eficacia cuando es capaz de integrar los objetivos individuales de los empleados dentro de los objetivos de la organización. Por otra parte, la planificación estratégica de los recursos humanos y el plan, que es uno de sus productos, aportan beneficios sustanciales a la gestión institucional al definir en horizontes de corto y de largo plazo, el nivel y las competencias necesarias del personal para contribuir al logro de los objetivos estratégicos de la organización.

\section{La planeación, el horizonte institucional}

Caldera (2005) considera que la planeación es la función que tiene por objetivo fijar el curso concreto de acción que ha de seguirse, estableciendo los principios que habrán de orientarlo, la secuencia de operaciones para realizarlo y las determinaciones de tiempo y números necesarios para su realización. Podemos considerar a la planeación como una función administrativa que permite la fijación de objetivos, políticas, procedimientos y programas para ejercer la acción planeada.

El mismo autor clasifica los planes que las organizaciones deben tener en cuenta al momento de llevar a cabo este proceso de la siguiente manera: (i) según la clase de plan: objetivos, políticas a seguir, procedimientos, métodos, programas y presupuestos; (ii) según los fines del plan: nuevos, vigentes y correctivos; y (iii) según el uso del plan: para usarse una sola vez o varias veces.

De esta manera, se tiene que la planeación en toda organización constituye el horizonte hacia donde se deben dirigir todos los esfuerzos a fin de tener una visión clara de lo que se desea; este proceso debe involucrar a todas las personas que participan en la institución, principalmente los directivos que deben tener bien claro el camino a seguir. 


\section{La planeación estratégica, un conjunto de acciones para lograr los objetivos}

Según Acle (1992), la planeación estratégica es un conjunto de acciones que deben ser desarrolladas para lograr los objetivos estratégicos, lo que implica definir y priorizar los problemas a resolver, plantear soluciones, determinar los responsables para realizarlos, asignar recursos para llevarlos a cabo y establecer la forma y periodicidad para medir los avances. Por su parte, Mintzberg y Brian (1993) consideran la planeación estratégica como el proceso de relacionar las metas de una organización, determinar las políticas y programas necesarios para alcanzar objetivos específicos en camino hacia esas metas, y establecer los métodos necesarios para asegurar que las políticas y los programas sean ejecutados.

Mintzberg y Brian (1993) establecen una serie de características de la planeación, las cuales entran a formar parte integral de la estrategia: debe ser conducida o ejecutada por los altos niveles jerárquicos, establece un marco de referencia para toda la organización, afronta mayores niveles de incertidumbre con respecto a otros tipos de planeación, generalmente, cubre amplios períodos (cuanto más largo el período, más irreversible será el efecto de un plan estratégico), y finalmente, su parámetro es la eficiencia.
Se tiene entonces que cuando se habla de planeación se hace referencia a un proceso formal para generar resultados articulados en la forma de un sistema integrado de decisiones; es decir, es la descomposición de un proceso en pasos claros y articulados asociados a un proceso racional. La planeación estratégica incluye liderazgo, conocimiento organizacional, competencia, estructura de la empresa, así como la formulación de estrategias maestras fundamentales en su aplicación temporal. Con ella, los administradores hacen coincidir los recursos de la organización con sus oportunidades de mercado a un largo plazo.

\section{Los objetivos de la planeación estratégica, guía para la puesta en marcha}

Caldera (2005) establece que el objetivo central de la planeación estratégica es lograr el máximo provecho de los recursos internos seleccionando el entorno donde se han de desplegar tales recursos, y la estrategia de despliegue de los recursos. Por ejemplo, se trata de encontrar un nicho de mercado que la empresa pueda atender mejor que los posibles competidores, donde, por lo tanto, la aplicación de los recursos resulte más provechosa que en otras circunstancias.

El mismo autor considera que otro objetivo de la planeación estratégica es analizarla 
como un medio fundamental para mostrar una actitud de cambio frente a lo que pueda pasar en el entorno, permitiendo un control continuo sobre el direccionamiento de la organización. Además, debe discernir a través de la gerencia estratégica entre cada uno de los factores que influyen en la empresa, permitiendo así elementos para una toma de decisiones estratégicas que faciliten una reacción positiva ante dichos factores y resaltando el papel que juegan las estrategias en el entorno de la globalización.

\section{La planificación estratégica de recursos humanos, una forma efectiva de identificar necesidades de personal}

Todas las personas que intervienen en la planificación estratégica esperan mejorar las organizaciones, en otras palabras, hacerlas más eficientes, más humanas, más racionales, más útiles y rentables para las sociedades y los propietarios, más satisfactorias para los miembros, más estables, más flexibles, o cualquier otra cosa que se necesite. Las oportunidades para mejorarlas son muy grandes. Cada transformación estructural que se genere podrá ser entonces el principio de un proceso que, de manera definitiva, genere grandes cambios en la estructura organizacional para colocar a la empresa en posición de competir y sobre- vivir frente a un entorno globalizado que demanda eficiencia y transparencia en el ejercicio de sus funciones.

Desde la perspectiva de las organizaciones públicas, la planeación estratégica de recursos humanos tiene un papel crucial si se toma en cuenta que los desafíos a los cuales debe responder son cruciales al interior de la misma.

La gestión estratégica de recursos humanos puede concebirse como una gran sombrilla que integra las prácticas de recursos humanos, las políticas y la filosofía con el objetivo de preparar a la organización para lograr sus metas estratégicas. Idealmente, estas prácticas y políticas deben formar un sistema capaz de atraer, desarrollar, motivar y entrenar la dotación de empleados necesaria para asegurar el efectivo funcionamiento de una organización.

La planeación estratégica de recursos humanos puede ser definida en términos amplios como el proceso de análisis de las necesidades de recursos humanos conforme cambian los entornos internos y externos de la organización, y la aplicación de la consiguiente estrategia proactiva para asegurar la disponibilidad de recursos humanos demandada por la organización.

Para Caldera (2005), la planeación de recursos humanos es el proceso de anticipar 
y prevenir el movimiento de personas hacia el interior de la organización, dentro de ésta y hacia fuera. Su propósito es utilizar estos recursos con tanta eficacia como sea posible, donde y cuando se necesiten, a fin de alcanzar las metas de la organización.

En este sentido, la planeación de recursos humanos, también denominada planificación de la plantilla o del personal, es un proceso que permite situar el número adecuado de personas calificadas en el puesto adecuado y en el momento adecuado.

Adicionalmente, se puede definir a la planeación de los recursos humanos como el sistema que permite ajustar la oferta de personal interna (empleados disponibles) y externa (empleados que se buscan o se han de contratar) a las vacantes que espera tener la organización en un periodo dado.

Se tiene entonces que la planificación, en consecuencia, consiste en definir unos objetivos concretos y diseñar los sistemas a seguir para conseguirlos, así como cuantificar los medios necesarios estableciendo unos plazos de tiempo determinados.

\section{El capital humano, motor de toda organización}

Porter (1990) argumenta que el personal de una organización puede aportar ventajas competitivas superiores (de difícil imitación) y de mucha flexibilidad para que ésta pueda enfrentar la competencia o desarrollar servicios diferenciados. Este autor estima que toma aproximadamente siete años duplicar una ventaja competitiva fundamentada en recursos humanos, y que mientras más difícil o costoso sea imitar la ventaja competitiva, más durable será la misma, por tanto, estas ventajas fundamentadas en la base de recursos humanos de las organizaciones representan una fuente duradera de difícil imitación. Cabe señalar, que al igual que cualquier otro recurso productivo, los recursos humanos pueden deteriorarse y las habilidades y conocimientos pueden convertirse en obsoletos y perder su potencial de contribución al rendimiento de la institución, por lo cual, se hace necesario mantener un programa de inversiones en actualización de conocimientos y desarrollo de habilidades para que este recurso no pierda valor (Dessler, 2001).

Por otro lado, directivos y ejecutivos públicos demandan cada vez más un giro articulado de los departamentos de recursos humanos para moverse de un valor percibido a través del entrenamiento de los empleados, hacia un valor añadido real percibido por los clientes o usuarios. En consecuencia, el plan estratégico de recursos humanos sería el instrumento de respuesta para que estos recursos y esfuerzos del área de personal se traduzcan en valores económicos reales para los 
clientes o usuarios externos; pero aún más, la contribución del análisis de puesto como eje fundamental de la planeación estratégica de recursos humanos, queda demostrada por el hecho de que una vez se hayan seleccionado los ocupantes de puestos, su desempeño se va a guiar por estas descripciones de puestos actualizadas, y de la misma forma, la evaluación de su desempeño será realizada comparando los estándares establecidos en su puesto con su actuación en el mismo.

\section{Objetivos de la planeación de recursos humanos, el norte}

Según Valle (1995), los objetivos básicos de la planeación de recursos humanos son: optimizar el factor humano de la empresa; asegurar en el tiempo la plantilla necesaria, cualitativa y cuantitativamente; desarrollar, formar y proporcionar al personal actual de acuerdo con las necesidades futuras de la empresa; motivar al factor humano de la empresa; mejorar el clima laboral; y contribuir a maximizar el beneficio de la empresa.

Se tiene claro entonces que planear los recursos humanos significa aunar esfuerzos de manera que se desarrollen las distintas cualidades del personal, teniendo en cuenta las distintas necesidades empresariales que coadyuven a mantener un clima acorde con las exigencias del mundo globalizado.

\section{Planeación de recursos humanos y la importancia que reviste}

Siguiendo a Milkovich y Boudreau (1994), la importancia de la planeación de los recursos humanos se manifiesta cuando las organizaciones se ven en la necesidad de proyectar puestos de trabajo, ubicar plantas, recortar personal o clausurar instalaciones de operación.

Se enumeran los porqués de la planeación de los recursos humanos desde la perspectiva de los autores:

i. Retener en calidad y cantidad: el éxito a largo plazo de una organización depende definitivamente en lograr tener las personas adecuadas en los puestos adecuados y en el momento adecuado. Los objetivos y estrategias definidos por la planeación de recursos humanos sólo tienen sentido cuando se dispone de personas con las capacidades, habilidades y ambición apropiadas para llevar a cabo estas estrategias. Una mala planificación de los recursos humanos puede traer a la empresa problemas graves a corto plazo.

ii. Prever los cambios: la necesidad de una planeación de recursos humanos se debe principalmente al gran desfase temporal que se presenta entre el reconocimiento de la necesidad de cubrir un puesto y el hallazgo de una persona apta para desempeñarlo. 
Una planeación de recursos humanos eficaz ayuda también a reducir la rotación de personal al mantener a los empleados informados acerca de las oportunidades de carrera dentro de la organización.

\section{La interrelación entre planificación estratégica empresarial y planificación estratégica de recursos humanos como una necesidad}

La planeación estratégica de la empresa identifica varios factores críticos para lograr el éxito de la organización a la vez que trata de encontrar el modo de que ésta se sitúe en mejor posición y esté en mejores condiciones para competir en el mercado. Para ello, el proceso de planeación ofrece: una formulación clara de la misión de la organización, un compromiso del personal con esa misión, una declaratoria expresa de las hipótesis de partida y un plan de acción ajustado a los recursos disponibles, incluida la dotación de personal capacitado y formado.

La planificación de recursos humanos contribuye significativamente al proceso de dirección estratégica, pues aporta los medios para alcanzar los resultados esperados del proceso de planificación. Las demandas y necesidades de recursos humanos derivan de la planeación estratégica y operativa, y luego son comparadas con las necesidades existen- tes; para ello se elaboran programas de reclutamiento, capacitación y reasignación.

La planeación de recursos humanos considera la aplicación del proceso básico de planificación a las necesidades de recursos humanos de una organización. Todo plan de recursos humanos, para que sea eficaz, debe basarse en los planes operativos a largo plazo de la organización. Su logro dependerá del grado en el que el departamento de recursos humanos pueda integrar la planificación efectiva de personal en el proceso de planificación global de la empresa.

Para integrar los recursos humanos a la planeación estratégica se puede:

i. Suministrar un FODA $^{4}$ de recursos humanos: los cambios constantes del entorno modifican el estado de la organización y es necesario que se conozca el ambiente social, demográfico y sindical, ya que influyen directamente en el ambiente interno como la organización del trabajo y de los empleados.

ii. Asegurar que los asuntos de recursos humanos se estudien en cada una de las cuatro etapas del proceso de decisión: objetivos, táctica, evaluación y elección.

\footnotetext{
4 Estudio diseñado para determinar fortalezas, oportunidades, debilidades y amenazas presentadas por la organización.
} 
iii.Alinear las prácticas de la gerencia de los recursos humanos en función de la táctica, y hacerlas coherentes.

El proceso de planificación de recursos humanos con base en competencias, una forma de evaluar los objetivos

Hablar del proceso de planificación de recursos humanos con base en competencias es tener conciencia de la importancia que reviste este departamento dentro de la organización; tal como lo mencionaba Puchol (2007), planificar el negocio es planificar el personal, de allí que se hace necesario elaborar una serie de estudios que identifiquen plenamente la situación en que se encuentra la organización para luego conocer qué aspectos la separan de lo que se espera en el largo plazo, lo que permitirá elaborar los planes requeridos.

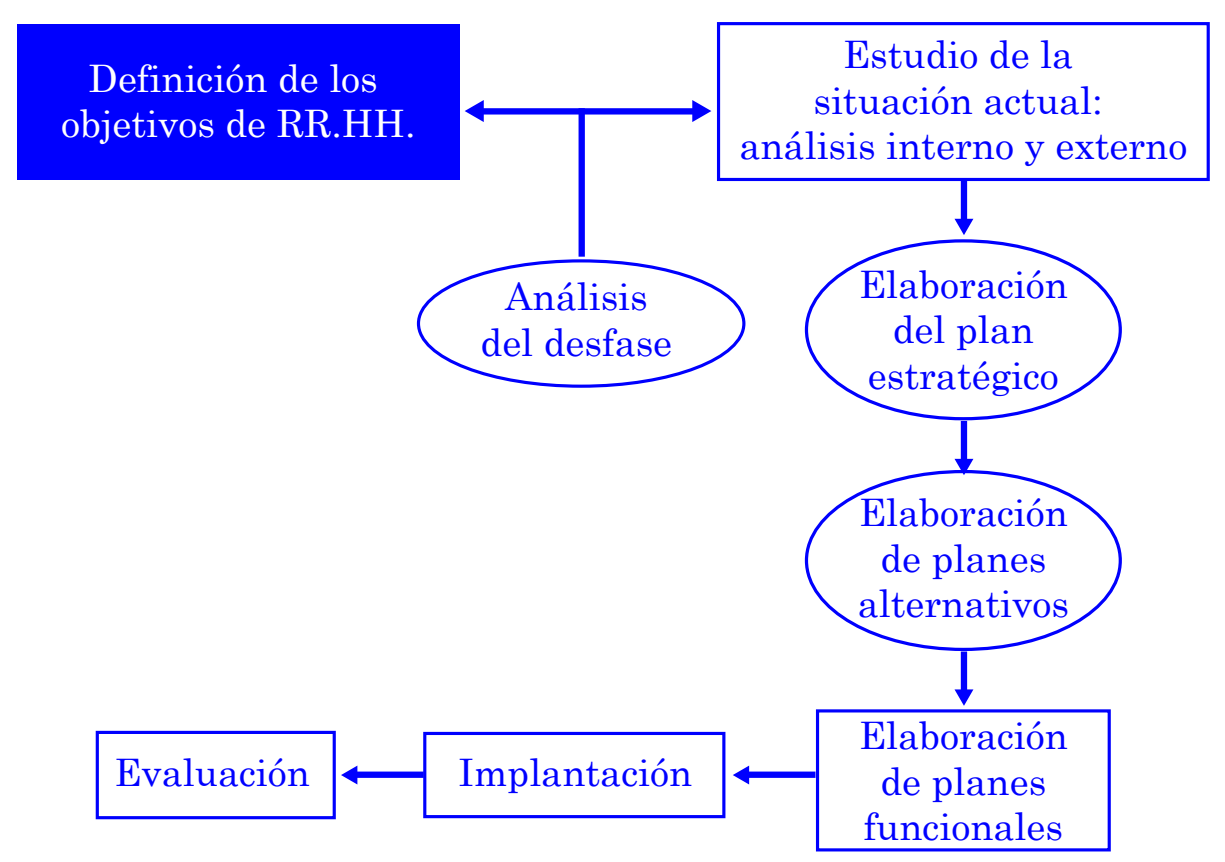

Figura 1. Proceso de planificación de recursos humanos.

Fuente: Nieves (s.f.) 
Tal como lo muestra la figura 1, el proceso de planificación de recursos humanos con base en competencias se considera como la forma efectiva para evaluar los objetivos de la sección, puesto que a través de éste se hace un análisis de la situación actual donde se estudian los aspectos internos y externos de la organización, se analiza el desfase entre lo existente y lo visionado para luego elaborar los planes estratégicos, alternativos y funcionales, así se procede a la implantación del plan para culminar en la evaluación, la cual se recomienda realizar en la medida que cambien las circunstancias.

En este sentido, y tal como lo establece Caldera (2005), la planeación de recursos humanos es el proceso que busca asegurar el óptimo aprovechamiento del personal con el que cuenta la empresa y proporcionar los recursos humanos relacionados con las necesidades futuras de la organización. Para alcanzar plenamente su objetivo, la planeación estratégica de recursos humanos debe considerar los factores del medio ambiente, es decir, la definición del entorno macro de la organización tomando en cuenta las características de la actividad económica a la cual pertenece la empresa, las acciones de gobierno que se emprenden en ese sector, el avance tecnológico, las cuestiones sociales $\mathrm{y}$, sobre todo, las características y penetración en el mercado de los competidores.
El mismo autor cree que la planeación debe involucrar aspectos como: identificar la misión y los objetivos institucionales; conocer con precisión la estructura y las funciones de la organización; comprender exactamente los programas y proyectos prioritarios o sustantivos de apoyo; establecer políticas, procedimientos y programas en materia de administración de recursos humanos; y precisar los requisitos que debe reunir el personal, entre otros, la información extra-institucional, estatus socioeconómico, nivel cultural y problemática familiar, así como la información intra-institucional. En términos de inventario de recursos humanos, se deben incluir las habilidades, el reclutamiento, la selección, la contratación, la inducción, el desarrollo, los sueldos y salarios, las relaciones laborales y los motivos de salida del personal. Por último, se deben elaborar diagnósticos y pronósticos del personal para conocer sus características y planear dotaciones o movimientos futuros.

\section{Las fases de la planificación estratégica de recursos humanos, una forma de visionar el camino}

Para tener un amplio conocimiento sobre cada una de las fases de la planificación estratégica de recursos humanos, Jiménez (2007) muestra los aspectos necesarios en cada una de ellas. De esta forma, se pueden 
distinguir las siguientes fases o etapas en el proceso de planificación:

a. Fase del análisis. La fase de análisis parte del conocimiento exhaustivo de la empresa. En esta fase se considerarán aspectos relacionados con la organización general actual, las grandes áreas de actividad, las funciones de las unidades que constituyen las áreas de actividad, las funciones de las categorías o grupos laborales que integran dichas áreas, las responsabilidades que corresponden a cada nivel estructural por áreas de actividad, así como las políticas y estrategias generales y específicas.

La materialización de esta fase, según el mismo autor, requiere disponer de la siguiente información: un organigrama general o básico de la empresa actualizado, los organigramas detallados de cada una de las grandes áreas, los manuales de funciones de las unidades organizativas que constituyen esas áreas, los manuales de funciones de las categorías que integran las áreas, la descripción de todos los puestos de trabajo actuales, los profesiogramas, un inventario de la totalidad de puestos de trabajo existentes, los resultados de las valoraciones de los actuales puestos de trabajo, los resultados de los principales ratios relativos a la plantilla, lo que corresponde al plan de empresa. b. Fase de previsión. El objetivo de esta fase es conocer la situación y necesidades de la empresa en el futuro, los cambios organizativos que se producirán y los derivados de la propia actividad empresarial o del sector. El desarrollo de esta fase implica el estudio de aspectos como son, conocer los organigramas previstos, analizar y describir los puestos de trabajo necesarios en ese futuro, valorar esos puestos, cuantificar las necesidades de nuevos puestos, preparar las fuentes de reclutamiento internas o externas, diseñar los sistemas idóneos de selección de ese personal, establecer los planes de formación y desarrollo de los nuevos puestos, preparar las fuentes de reclutamiento internas o externas, diseñar los sistemas idóneos de selección del personal y establecer los planes de formación y desarrollo de los nuevos puestos.

c. Fase de programación. En esta fase se establecen la metodología y procedimiento para la realización de estudios indicados en el punto anterior, la prevención de las distintas actividades en temporalización y el equipo que llevará a cabo toda la planificación.

d. Fase de realización. Es la fase en la que se ponen en práctica todas y cada una de las actividades indicadas en las fases anteriores. 
e. Fase de control. Es el análisis, en el tiempo, de las desviaciones que se van produciendo a lo largo del desarrollo del plan.

f. Fase de presentación de resultados. Información referente a los puestos de trabajo. La planificación eficaz de los recursos humanos hace adecuada descripción de los puestos de trabajo de la empresa.

De esta manera, una descripción de puestos debe contemplar cuatro puntos básicos como son: la descripción genérica del puesto de trabajo; la descripción cuantitativa del puesto de trabajo: medios y recursos a utilizar, así como resultados a obtener; la situación del puesto de trabajo en la estructura organizativa; y la situación interna del puesto de trabajo: especificación referente al desempeño laboral.

\section{El análisis FODA como herramienta fundamental para conocer el recurso humano de la organización}

Indiscutiblemente, si se desea conocer la situación actual del recurso humano con el objeto de llevar a cabo una planificación estratégica, se hace indispensable realizar el análisis FODA. Este estudio debe contemplar el análisis interno y externo del talento humano en el que se determinan las fortalezas, oportunidades, debilidades y amenazas que presenta actualmente la organización.
En este sentido, Granjo (2008) expone las características que este análisis debe presentar, en el cual se deben mostrar los puntos fuertes y débiles de la organización para luego establecer el plan estratégico requerido.

\section{El análisis externo, una constituyente de las oportunidades y amenazas}

El diagnóstico del entorno ayuda a obtener una mejor comprensión del contexto en el que se toman y deberán tomarse decisiones de recursos humanos. En este sentido, son varias las cuestiones a considerar, de las que se pueden nombrar las condiciones del mercado de trabajo. Aspectos tales como el grado de envejecimiento de la población activa, corrientes migratorias, participación de colectivos minoritarios en la composición de la fuerza laboral, nivel de desempleo, incorporación de la mujer al trabajo, nivel de formación, etc., son aspectos de obligada consideración para la planificación de los recursos humanos.

Por otra parte, la incorporación de la mujer al mercado laboral plantea serias exigencias a la gestión de los recursos humanos. De un lado, la eliminación de barreras aún presentes en el proceso de afectación del denominado sexo débil, y por otro, la 
instauración de procesos que compatibilicen sus posibilidades de carrera profesional con la labor que la madre naturaleza le encomendó: maternidad y primeros cuidados.

La evolución cuantitativa y cualitativa de determinadas categorías profesionales en el mercado laboral alertará sobre una posible escasez futura de ciertos perfiles. Es ésta la curiosa paradoja del mercado laboral, pues mientras persiste el desempleo existen puestos vacantes por inadecuación a los perfiles deseados.

Tal como lo manifiesta Granjo (2008), es importante que la organización identifique su mercado laboral relevante, éste, en su sentido más amplio sin referirlo a ningún puesto de trabajo concreto, vendrá marcado por el ámbito geográfico, la naturaleza del negocio y por la situación competitiva de la firma.

En este sentido, la incidencia del Estado en el nivel de empleo como usuario, por una parte, y como protector de la clase obrera a través de la legislación socio-laboral, por otra, deberá ser tenida en cuenta. En este último aspecto, cobra especial relevancia la flexibilidad que otorga la normativa a las empresas para que éstas adquieran, usen y prescindan de la fuerza de trabajo.
Cuestiones tales como la diversidad contractual en materia laboral, regulación de salarios y horarios de trabajo, reglamentos sobre horas extraordinarias, compensaciones por despidos y algunas otras son de obligada consideración.

La situación de la industria o sector es otro aspecto a tener en cuenta en el análisis externo, pues el grado de competitividad existente en el entorno específico de la organización y su previsible evolución nos alertará e informará sobre las líneas a seguir en la planificación de recursos humanos. Un giro brusco o acentuación de la estrategia genérica de alguno de los principales competidores afectará indudablemente al proceso. Esta idea permite introducir una nueva dimensión de la lucha competitiva: el poder de atracción de las empresas con relación a los empleados potenciales.

El grado de innovación tecnológica que presida el ambiente exterior determinará la demanda de trabajo a futuro. Téngase en cuenta que los cambios tecnológicos, incluyendo la robótica y la automatización de oficinas, inciden notoriamente en los índices de productividad así como en la creación y eliminación de puestos de trabajo. 


\section{El análisis interno, una constituyente de las fortalezas y debilidades}

Granjo (2008) establece que el contexto no sólo viene determinado por el entorno de la organización; en él también participan en su formación ciertos factores localizados en el propio seno de la organización. Así las cosas, el principal factor causal de la planificación de recursos humanos reside en la alternativa estratégica elegida por la compañía para alcanzar sus objetivos, la cual habrá sido desglosada en planes para cada una de las funciones o subsistemas de la organización como son producción, finanzas, marketing, etc.

El estado del sistema productivo, así como las inversiones previstas ligadas a él, son determinantes en los indicadores de productividad probables en un tiempo más o menos próximo; indicadores determinantes de la demanda de empleo en este ámbito son el número de rechazos en relación directa y la productividad alcanzada en relación inversa.

Otro aspecto a considerar es el contenido del trabajo, se trata de realizar una investigación sistemática centrada en los puestos y procesos de trabajo, tratando de prever los requisitos que garanticen un eficaz desempeño.
Con respecto a la estructura y cultura organizativa, se pretende determinar cómo afecta la estructura y la cultura de una organización al logro de sus objetivos. Su análisis exige cuestionar el número total de personas, sus creencias, normas y valores, su composición por categorías y su distribución por departamentos. Esta cuestión es de obligada naturaleza a la luz de posibilidades de fusión o absorción.

Las competencias y motivación de los recursos actuales mantienen relaciones directamente proporcionales con la productividad $\mathrm{y}$, por tanto, inversamente proporcionales con las necesidades futuras de personal. La identificación de las competencias y potenciales individuales pueden ser tomados de la entrevista de evaluación anual, si es que ésta está institucionalizada. El inventario de habilidades constituye un instrumento útil para estos efectos, pues describe el nivel de conocimientos, competencias y habilidades disponibles en el seno de la organización.

La rotación, sobre todo la rotación no prevista, constituye uno de los factores que en mayor grado dificultan la tarea de planificar el subsistema de personal (Tyson y York, 1989). Resultan por ello necesarias las estimaciones del grado de rotación que padecerá la empresa en el horizonte temporal contemplado en función de las diversas categorías profesionales. El análisis de la pirámide de 
edades se configura como una herramienta básica para la estimación de la rotación previsible.

El absentismo laboral es otro de los determinantes de las necesidades de personal, por ello, conviene hacer acopio de datos a efectos de proyección, desglosándolos en sus dos vertientes principales: quiere, pero no puede, y puede, pero no quiere.

Las transferencias internas, por su parte, implican aspectos de rotación en la unidad exportadora y de afectación en la unidad importadora. Especial importancia juegan las promociones o ascensos del personal, por constituir éstas una de las modalidades más frecuentes dentro de esta categoría de movimientos, además de dar lugar a impactos psicosociales intensos (estrés, conflictos, etc.).

Una vez analizado el ambiente, se inicia el proceso de la elaboración de las estimaciones; como criterio de elección primario, se puede tener en cuenta que las organizaciones con entornos estables preferirán hacer uso de técnicas cuantitativas, mientras que aquellas que afrontan entornos rápidamente cambiantes, se inclinarán por la aproximación cualitativa.

Luego de establecer un análisis de la situación actual de la empresa, se establece cuál es el desfase existente entre lo que se tiene y lo que se quiere o se desea alcanzar en un futuro para luego desarrollar lo que es el plan estratégico de recurso humano.

\section{El plan estratégico del recurso humano, mecanismo de éxito organizacional}

Benito (2003) considera que crear un plan estratégico de recursos humanos es una decisión muy seria que requiere un tiempo previo de reflexión sobre su oportunidad y posibilidades de éxito; que hacer un proyecto de esta naturaleza conlleva un doble compromiso, por una parte, con la dirección de la compañía y con ésta en su conjunto, y por la otra, un compromiso muy próximo y directo con la propia función de recursos humanos y con el equipo de profesionales que la desarrolla. Sin embargo, es una tarea que hay que desarrollar, debido a que los beneficios son mayores a los retos propuestos, ya que se logran modelos estructurales en función de los distintos grupos de clientes que acredita un buen desempeño laboral.

De esta manera, concluye al autor, que fueron esencialmente cinco razones que lo movieron a desarrollar la iniciativa para afrontar el reto propuesto: concretar la contribución esperada de la función de recursos humanos al logro de los objetivos estratégicos de calidad, rentabilidad y servicio al 
cliente de la organización; renovar el compromiso de la dirección de recursos humanos como unidad de servicio a los objetivos estratégicos de la empresa y al desarrollo profesional de los empleados; dotar al equipo del departamento de recursos humanos de un marco global que garantizara la orientación común y la coherencia del trabajo de todos y cada uno de sus miembros; fijar y transmitir objetivos concretos de gestión al equipo de recursos humanos e impulsar su eficacia; y dar visibilidad al valor añadido que la función de recursos humanos es capaz de aportar al negocio, en el presente y futuro de la empresa y publicar el carácter estratégico de su misión.

Para dar cumplimiento al plan estratégico, se hace necesario tener en cuenta los siguientes aspectos: imaginar todos los caminos posibles desde la situación actual hasta la situación ideal, estudiar desde todos los puntos de vista posibles (técnicos, humanos, económicos, etc.) todas las soluciones propuestas, eliminar aquellas que parezcan no factibles y seleccionar las que sí parezcan llevar a los objetivos; así se elige la opción (a priori) más adecuada para el plan estratégico de recursos humanos.

Granjo (2008) considera que el plan debe incluir la forma en que se van a adquirir las competencias que actualmente no se disponen y que son necesarias; la forma en que se van a desarrollar las competencias que ya dispone la empresa, pero se van a necesitar a niveles distintos; la forma en que se van a activar las competencias que se poseen para que se pongan en práctica; y la forma en que se van a inhibir ciertas competencias no deseadas.

\section{Los planes alternativos, una alternativa del plan estratégico}

Una vez se ha establecido que el proyecto es viable debe decidirse por una línea de actuación. Deben fijarse prioridades, señalar plazos, concretar las personas que han de desarrollar las acciones y el momento de comenzarlas, asegurar el suministro de recursos humanos suficientes y técnicamente adecuados. De esta forma, es recomendable elaborar planes alternativos para posibles eventualidades.

i. Ejecución del plan. Seguir escrupulosamente el camino trazado por el plan: contratación, promociones, formación, etc.

ii. Seguimiento. A medida que se desarrolla el plan deben existir controles que informen a la dirección de los riesgos de desviación o de incumplimiento de plazos, con los que se desencadenarán las medidas necesarias para corregirlo. 


\section{Conclusiones}

A modo de reflexión final, se puede decir que la planificación estratégica de recursos humanos y el plan, que es uno de sus productos, aportan beneficios sustanciales a la gestión institucional al definir en horizontes de corto y de largo plazo, el nivel y las competencias necesarias del personal para contribuir al logro de los objetivos estratégicos de la organización.

El papel de la planificación de recursos humanos no es el de actuar como componente reactivo del plan estratégico solamente, también puede señalar nuevas oportunidades y fortalezas fundamentadas en los recursos humanos actuales y futuros de la organización, además, permite identificar las limitaciones de recursos humanos que debiliten la viabilidad del plan estratégico

Implementar un plan estratégico del recurso humano para la organización implica necesariamente cumplir una serie de etapas y procesos que le permitirán a la organización desarrollar un análisis de la situación actual y confrontarlo con los objetivos que se propone y que se han visionado en el largo plazo.

La forma como se debe implementar la planificación estratégica del recurso humano contempla varias fases, como son: la definición de los objetivos de recurso humano, el estudio de la situación actual, el análisis del desfase, la elaboración de los planes estratégicos, alternativos y funcionales, y por último, la implementación y evaluación del proceso que contempla la definición de la plantilla de manera cuantitativa y cualitativa, culminando en la elaboración de un plan de carrera.

Los requerimientos o necesidades netas de personal no representan únicamente insuficiencias o excedentes del nivel y calificaciones de recursos humanos, sino que sus resultados demandan un programa de acciones para que este nivel de recursos humanos se ajuste en forma consistente con los objetivos del plan estratégico, de ahí pueden ser necesarias políticas de reclutamiento y contratación o políticas de reducción de personal, dependiendo de los resultados del análisis.

Finalmente, la planificación estratégica de los recursos humanos es una actividad propia de las organizaciones que alcanza su máxima eficacia cuando es capaz de integrar los objetivos individuales de los empleados dentro de los objetivos de la organización, constituyéndose en una forma efectiva de identificar necesidades de personal. 


\section{Referencias}

Acle, A. (1992). Planeación estratégica y control total de la calidad. México: Grijalbo.

Benito, C. (2003). Diseño y aplicación de un plan estratégico de recursos humanos. La experiencia práctica de DHL Internacional España. Capital humano, 162(16): 42-48.

Caldera, R. (2005). Planificación estratégica de recursos humanos: Conceptos y teorías. Editorial eumed.net

Dessler, G. (2001). Administración de personal. México: Pearson.

Granjo, J. (2008). Cómo hacer un plan estratégico de recursos humanos. La Coruña: Netbiblo.

Jiménez, P. (2007). Manual de recursos humanos. Madrid: ESIC.
Milkovich, G. y Boudreau, J. (1994). Dirección y administración de recursos humanos. México: Addison-Wesley Iberoamericana.

Mintzberg, H. y Brian, J. (1993). El proceso estratégico. México: Prentice Hall Hispanoamericana.

Nieves, E. (s.f.). Generalidades de la planificación estratégica. Recuperado de http://slideplayer.es/slide/4848693/

Porter, M. (1990). The Competitive Advantage of Nations. New York: Free Press.

Puchol, L. (2007). Dirección y gestión de recursos humanos. Madrid: Díaz de Santos.

Tyson, S. y York, A. (1989). Administración de personal. México: Trillas.

Valle, R. (1995). La gestión estratégica de los recursos humanos. México: Addison-Wesley Iberoamericana. 